\section{Clinical trials recruiting}

With this issue Neurology introduces a section in which investigators may list IRB-approved notification for clinical trials. In the past Neurology has had advertisements for clinical trials scattered throughout the journal. Now these will be included in a single section similar to the classifieds, with full page, partial page, and text listings organized by disease. Each notice will include a brief description of the study and its sponsorship as well as contact information. Steven M. Greenberg, MD, Editor, and Merit Cudkowicz, MD, Co-Editor, will address issues as they arise during the section's introduction and evolution.

see page $61 \mathrm{~A}$

The accompanying editorial by Steven Schwid and John Marler emphasizes that most clinical trials have problems with recruitment that delay or prevent their completion. These issues are of particular concern for NINDS, which currently provides support for approximately 120 trials of therapeutic interventions requiring nearly 10,000 patients per year. Investigators need to utilize a variety of recruitment strategies to speed enrollment, to ensure that enrolled patients provide generalizable results, and to enhance minority recruitment. Neurology will promote this section with a banner on the cover of the journal, a listing in the Table of Contents, and links on the journal home page (www.neurology.com). With a circulation to most neurologists worldwide and broader exposure through the journal Web site, this may grow to be a particularly efficient mechanism to inform clinicians and patients about research opportunities, developing physician networks, and patient resources.

see page 4

\section{Paroxysmal eyelid movements in generalized epilepsy}

Camfield et al. describe 19 children and adults (8 to 38 years) with persistent frequent, nonepileptic paroxysmal eyelid movements and well-controlled photosensitive generalized epilepsy (illustrated with on-line video). Paroxysmal eyelid movements were a source of diagnostic confusion but direct examination and video during EEG recording distinguished the attacks from absence seizures. There is an association between paroxysmal eyelid movements and photosensitive generalized epilepsy, which may create diagnostic confusion.

see page 40

\section{Cerebral microbleeds and left ventricular hypertrophy}

In a brain gradient-echo MRI-echocardiography correlation study, Lee et al. found that left ventricular mass index is significantly associated with increased grade of cerebral microbleeds. The authors suggest that microbleeds are an indication of end-organ damage induced by chronic hypertension.

see page 16

"Incidental The accompanying editorial by microbleeds should Jonathan Rosand notes that since all no longer be disregarded as 'incidental.' They can constitute a diagnosis and call for treatment." brain hemorrhages leave permanent hemosiderin deposits, T2*-weighted $M R I$ provides a lifetime record of hemorrhages. Catastrophic, symptomatic hemorrhagic stroke is just the tip of the iceberg. Microbleed location on MRI can reliably distinguish between the presence of cerebral amyloid angiopathy (CAA) and the vasculopathy of longstanding hypertension. The data of Lee et al. suggest that microbleed burden in the deep gray and infratentorial brain regions reflects disease chronicity and severity. While there is little at present to offer patients with CAA other than avoidance of anticoagulation, patients with hyperintensive vasculopathy are likely to benefit from careful blood pressure reduction. The findings of hypertensive vasculopathy on the basis of T2*-weighted MRI imply inadequately treated blood pressure.

see page 6

\section{Anti-GluR3 antibodies are not frequent in Rasmussen's encephalitis}

A role for antibodies to GluR3 in the pathogenesis of Rasmussen's encephalitis (RE) has become accepted. Watson et al. question this view. In a comprehensive study they were unable to confirm the presence of antibodies to GluR3 in 30 patients with RE, or 49 with intractable epilepsy, suggesting that such antibodies are uncommon in these conditions.

see page 43 


\section{Copper deficiency myelopathy}

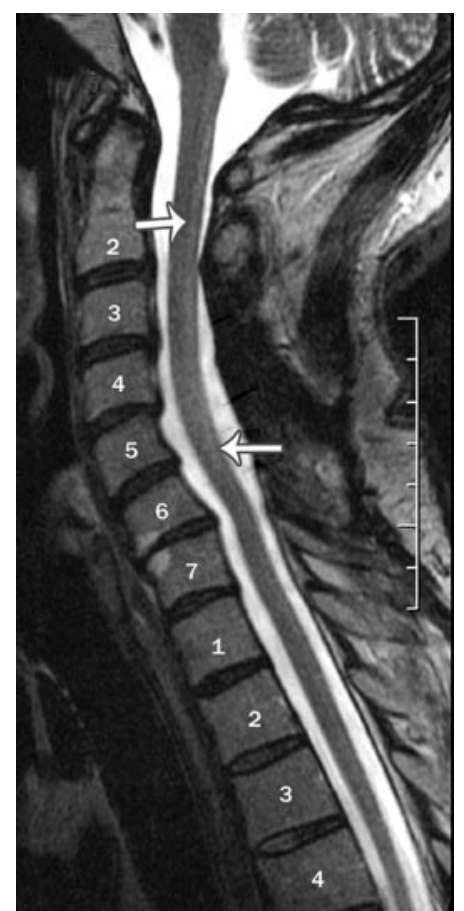

Cervical spinal cord MRI showing diffuse signal abnormality involving the central posterior aspect of the cord.

Kumar et al. report 13 patients with a syndrome resembling subacute combined degeneration linked to severe copper deficiency. Serum vitamin B12 levels were normal. The most characteristic presentation was a myelopathy with gait difficulty secondary to dorsal column dysfunction and lower limb spasticity. Copper supplementation prevented further neurologic deterioration, but the actual improvement was variable. In most patients the etiology of the hypocupremia was unclear.

\section{Self-treatment of benign paroxysmal positional vertigo (BPPV) of the posterior semicircular canal}

Radtke et al. found that self-treatment with a modified Epley's procedure was more effective than selftreatment with a modified Semont maneuver in 70 patients with posterior semicircular canal ( $95 \%$ vs $58 \%$ response rate after 1 week).

see page 150

The accompanying editorial by Joseph M. Furman and Tim Hain notes that BPPV is the most common cause of episodic vertigo. BPPV is caused by loose particles derived from the utricular maculae that are free-floating in the long arm of the posterior semicircular canal. They discuss the role of selftreatment for BPPV, concurring with Radtke et al. that selftreatment for BPPV may be beneficial for patients with frequent recurrences of BPPV. Since the recurrence rate for BPPV is approximately $50 \%$ in 4 to 5 years, particle repositioning is likely to become part of the routine management.

see page 8

\section{Clinical and MRI predictors of cognitive decline in aging and AD}

Adak et al. applied statistical models to 13 years of MRI and clinical longitudinal data acquired from 258 cognitively intact or questionably demented elderly. Data from MRI volumes (total white matter changes, \% ventricular and CSF volume) could predict rates of cognitive decline even in normal subjects. 


\section{Neurology}

July 13 Highlights

Neurology 2004;63;2-3

DOI 10.1212/WNL.63.1.2

\section{This information is current as of July 12, 2004}

\section{Updated Information \&}

Services

Permissions \& Licensing

Reprints including high resolution figures, can be found at: http://n.neurology.org/content/63/1/2.full

Information about reproducing this article in parts (figures,tables) or in its entirety can be found online at:

http://www.neurology.org/about/about_the_journal\#permissions

Information about ordering reprints can be found online:

http://n.neurology.org/subscribers/advertise

Neurology ${ }^{\circledR}$ is the official journal of the American Academy of Neurology. Published continuously since 1951, it is now a weekly with 48 issues per year. Copyright . All rights reserved. Print ISSN: 0028-3878. Online ISSN: 1526-632X.

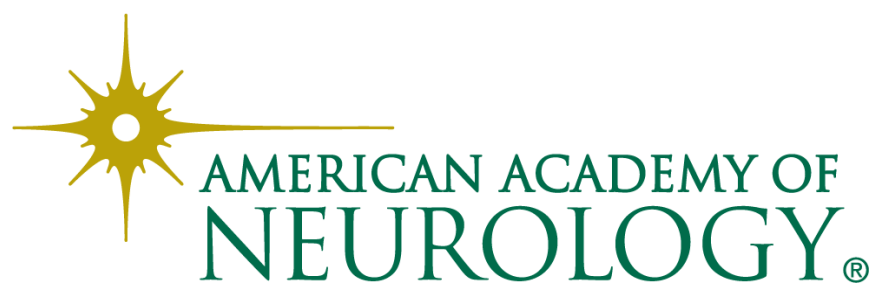

